\title{
Altered Intrinsic Coupling between Functional Connectivity Density and Amplitude of Low-Frequency Fluctuation in Mild Cognitive Impairment with Depressive Symptoms
}

\author{
Xiaozheng Liu $\mathbb{D},{ }^{1}$ Jiuzun Chen, ${ }^{1}$ Bangli Shen, ${ }^{1,2}$ Gang Wang, ${ }^{3}$ Jiapeng Li, ${ }^{3}$ Hongtao Hou, ${ }^{3}$ \\ Xingli Chen, ${ }^{3}$ Zhongwei Guo $\left(\mathbb{1 0},{ }^{3}\right.$ and Chuanwan Mao $\mathbb{1}^{1}$ \\ ${ }^{1}$ Department of Radiology, China-USA Neuroimaging Research Institute, The Second Affiliated Hospital and Yuying Children's \\ Hospital, Wenzhou Medical University, Wenzhou, Zhejiang 325027, China \\ ${ }^{2}$ Department of Pain, The Second Affiliated Hospital and Yuying Children's Hospital, Wenzhou Medical University, Wenzhou, \\ Zhejiang 325027, China \\ ${ }^{3}$ Tongde Hospital of Zhejiang Province, Hangzhou, Zhejiang 310012, China
}

Correspondence should be addressed to Zhongwei Guo; guozw1977@aliyun.com and Chuanwan Mao; maocwwz@163.com

Received 4 December 2017; Revised 6 March 2018; Accepted 7 May 2018; Published 29 May 2018

Academic Editor: Claudio A. Mastronardi

Copyright (C) 2018 Xiaozheng Liu et al. This is an open access article distributed under the Creative Commons Attribution License, which permits unrestricted use, distribution, and reproduction in any medium, provided the original work is properly cited.

Neuroimaging studies have demonstrated that major depressive disorder increases the risk of dementia in older individuals with mild cognitive impairment. We used resting-state functional magnetic resonance imaging to explore the intrinsic coupling patterns between the amplitude and synchronisation of low-frequency brain fluctuations using the amplitude of low-frequency fluctuations (ALFF) and the functional connectivity density (FCD) in 16 patients who had mild cognitive impairment with depressive symptoms (D-MCI) (mean age: 69.6 \pm 6.2 years) and 18 patients with nondepressed mild cognitive impairment (nDMCI) (mean age: $72.1 \pm 9.7$ years). Coupling was quantified as the correlations between the ALFF values and their associated FCDs. The results showed that the ALFF values in the D-MCI group were higher in the left medial prefrontal cortex (mPFC) and lower in the right precentral gyrus (preCG), and the FCD values were higher in the left medial temporal gyrus (MTG) than those in the $\mathrm{nD}-\mathrm{MCI}$ group. Further, correlation analyses demonstrated that, in the $\mathrm{D}$-MCI group, the mPFC was negatively correlated with the MTG. These findings may relate to the characteristics of mood disorders in patients with MCI, and they offer further insight into the neuropathophysiology of MCI with depressive symptoms.

\section{Introduction}

Mild cognitive impairment (MCI) is a neurological disorder that is associated with minimal cognitive impairments that are beyond those expected based on a person's age and education, but the changes are not severe enough to interfere with daily living, and they do not meet the criteria for dementia [1]. The conversion rate from MCI to dementia is approximately $12-16 \%$ per year [2]. Depression is a common symptom among individuals with MCI, with a prevalence of $32 \%$ [3].

Gao et al. [4] reported that depression was a major risk factor for the incidences of dementia and MCI. Further, recent meta-analyses showed that depressive symptoms increase the risk of MCI progressing to dementia and that depressive symptoms predict conversion from any type of MCI to all-cause dementia [5]. Neuroimaging studies have demonstrated that structural abnormalities in several brain regions are involved in the pathological process of depressive symptoms in MCI. Xie et al. [6] reported that depressive symptoms in MCI are related to grey matter volume loss in several brain regions, including the dorsal cingulate cortex, orbitofrontal cortex (OFC), ventromedial prefrontal cortex (vmPFC), posterior middle temporal gyrus (pMTG), and insula. Sacuiu et al. and Gonzales et al. [7, 8] also reported that MCI with depressive symptoms showed increased cortical atrophy in the anterior cingulate and the frontal lobe. Studies have also shown that, compared with those with 
nondepressed MCI (nD-MCI), patients with MCI and depression (D-MCI) have more white matter atrophy and white matter microstructural disruptions in the frontal, parietal, and temporal brain regions, especially in the hippocampal cingulum and fornix tracts [9-11]. Recently, researchers have used resting-state functional magnetic resonance imaging (R-fMRI) technology to study the functional changes in the brains of people with D-MCI. By analysing functional connectivity (FC), Zheng et al. [12] reported that, compared with $\mathrm{nD}-\mathrm{MCI}, \mathrm{D}-\mathrm{MCI}$ was associated with higher effective connectivity between the right amygdala and the right lingual gyrus, right calcarine gyrus, and bilateral supplementary motor areas. Xie et al. [13] reported that, for people with MCI, scores on the Geriatric Depression Scale were positively correlated with functional connectivity in the network connecting the bilateral posterior cingulate cortex (PCC), middle temporal gyrus, and left dorsolateral prefrontal cortex (DLPFC). By analysing the amplitude of low-frequency fluctuations (ALFF), Li et al. [14] found that abnormal ALFF values in the left inferior frontal gyrus and left precuneus could effectively differentiate nD-MCI from D-MCI. Currently, the preliminary results showed that the affective network and the default mode network might be simultaneously damaged in patients with D-MCI.

FC and ALFF are two fundamental fMRI parameters. FC characterises the degree of synchronisation between lowfrequency fluctuations in the resting brain and requires the definition of a relation (e.g., Pearson correlation) between the features of different voxels. Therefore, FC describes network properties, while the ALFF represents the amplitude of resting-state spontaneous brain activity by calculating the voxel-wise total power of a given fMRI time course within the low-frequency band [14]. These two measures have been shown to have a close relationship [15-17] and to be altered within the affective network and the default mode network in both MCI and major depressive disorder [12, 13, 18]. Compared with healthy elderly subjects, AD and MCI patients show absent FC density-ALFF coupling in the anterior and posterior cingulate cortex and the temporal cortex [15]. Similar methods have been used to observe changes in functional brain features in erythaematosus and epilepsy patients $[16,17]$. Thus, we believe that the coupling patterns between these two parameters may provide a new measure that can help us understand the underlying neuropathophysiology of D-MCI and enhance its identifiability by resting-state fMRI.

We, therefore, utilised the measures of ALFF and FC density (FCD), a global and voxel-wise measure of FC, to investigate alterations in amplitude-connectivity coupling in D-MCI. Given the common differences that have been reported in the brain networks of patients with D-MCI [13-17], we hypothesised that those with D-MCI would have altered brain fluctuations in brain regions associated with cognitive and emotional regulation.

\section{Materials and Methods}

2.1. Patients. Eighteen patients with $\mathrm{nD}-\mathrm{MCI}$ and 16 with $\mathrm{D}$ MCI were recruited from the outpatient department of Tongde Hospital in Zhejiang Province, China, from July
2013 to August 2016. The study was approved by the local ethics committee, and all participants gave their written informed consent before the MR scanning. All participants were right-handed, and the groups did not significantly differ in age, sex, or years of education.

A diagnosis of MCI includes memory impairment that does not meet the criteria for dementia. The criteria for MCI [19] were as follows: (a) impaired memory performance on a normalised, objective verbal memory test; (b) recent history of symptomatic worsening in memory; (c) normal or near-normal performance (score $>24$ ) on the MiniMental State Examination (MMSE), as well as on the activities of daily living scale (score $>24$ ); (d) a global rating of 0.5 on the clinical dementia rating scale; and (e) the absence of dementia.

Depressive symptoms were identified by professional psychiatrists according to the Diagnostic and Statistical Manual of Mental Disorders, fourth edition [20]. The severity of depressive symptoms was evaluated using two clinical scales: the Hamilton Depression Rating Scale (HAMD) [21] and the Neuropsychiatric Inventory (NPI) [22]. We considered HAMD scores $\geq 7$ and NPI scores $\geq 4$ in the depression domain to be clinically significant [23].

Patients were excluded if they had a history of psychiatric disorders, alcohol or substance abuse/dependence during the prior five years, MMSE scores $<24$, a history of neurological disease, MRI contraindications, or unstable chronic medical conditions.

2.2. MRI Scan. All scanning was collected using an 8channel head coil in a 3T Siemens scanner (Siemens Magnetom Verio; Siemens Medical Systems, Erlangen, Germany) at Tongde Hospital in Zhejiang Province. All patients were asked to keep their heads still and their eyes closed during image acquisition. T1-weighted highresolution anatomical images were acquired using a 3D magnetisation-prepared rapid gradient echo sequence with the following parameters: repetition time $=1900 \mathrm{~ms}$, echo time $=3.44 \mathrm{~ms}$, inversion time $=900 \mathrm{~ms}$, flip angle $=9^{\circ}, 128$ sagittal slices, field of view $=256 \mathrm{~mm}$, and slice thickness $=1 \mathrm{~mm}$. Functional resting-state fMRI images were acquired using a $\mathrm{T}_{2}{ }^{*}$-weighted echo-planar imaging sequence with the following parameters: 33 axial slices, thickness $/$ gap $=4.8 / 0 \mathrm{~mm}$, in-plane resolution $=3.4 \times 3.4 \mathrm{~mm}^{2}$, repetition time $=2000 \mathrm{~ms}$, echo time $=30 \mathrm{~ms}$, flip angle $=90^{\circ}$, and field of view $=200 \mathrm{~mm}$. Each condition lasted for $6 \mathrm{~min}$ $40 \mathrm{~s}$, and 200 functional volumes were obtained.

2.3. Data Preprocessing. All fMRI data were preprocessed using SPM8 (http://www.fil.ion.ucl.ac.uk/spm) and Data Processing Assistant for Resting-State fMRI (http://www. restfmri.net). The first ten time frames were discarded to ensure an MR steady state. The preprocessing steps comprised slice-timing correction for interleaved acquisitions, 3D motion correction, linear drift removal, spatial smoothing with a Gaussian smoothing kernel (full width at half maximum $=6 \mathrm{~mm}$ ), and spatial normalisation to the standard Montreal Neurological Institute (MNI) brain space with a resampling resolution of $3 \times 3 \times 3 \mathrm{~mm}^{3}$. Subjects were 
discarded if head motion exceeded $1.5 \mathrm{~mm}$ translation in $\mathrm{x}, \mathrm{y}$, or $\mathrm{z}$ directions or $1.5^{\circ}$ of maximum rotation about the three axes. All remaining smoothed images were filtered using a typical temporal bandpass $(0.01-0.08 \mathrm{~Hz})$ to reduce lowfrequency drift and physiological high-frequency respiratory and cardiac noise. Linear trends were also removed within each time series.

2.4. ALFF Calculation. ALFF was calculated using the REST software (http://www.restfmri.net). Briefly, for a given voxel, the time series was first converted to the frequency domain using a fast Fourier transform. The square root of the power spectrum was computed and then averaged across $0.01-0.08 \mathrm{~Hz}$. This averaged square root was taken as the ALFF at the given voxel [24]. Then, the ALFFs were standardised by dividing by the whole-brain average of the ALFF at each voxel, which measures the absolute strength or intensity of spontaneous low-frequency oscillations.

2.5. FCD Calculation. We performed voxel-based wholebrain correlation analysis on the preprocessed R-fMRI data, as has been well described in previous studies [25]. Pearson's correlation coefficients $(r)$ were computed between the time series for all pairs of grey matter voxels within a grey matter mask. The FCD for a given voxel was calculated as the sum of the significant connections between a given voxel and all other voxels by thresholding each correlation at $r>0.25$. Finally, to improve the normality of the data, the voxelwise FCD values were transformed into a $Z$-score map using a Fisher $Z$ transformation. Because of the uncertain interpretation and the detrimental effects on test-retest reliability, only positive correlations were considered in the FCD calculations.

2.6. Statistical Analysis. To examine ALFF and FCD differences between the groups, a two-sample $t$-test was performed between the two groups using REST. The 3dClustSim program, which is based on Monte Carlo simulation and implemented in AFNI (http://afni.nimh.nih.gov), was used for multiple comparison correction [26]. The statistical threshold was set at $P<0.005$ and cluster size $>28$ voxels, which corresponded to a corrected $P<0.05$.

2.7. Coupling of ALFF and FCD. To test the proposal that alterations in amplitude-connectivity coupling in D-MCI and, thus, to differentiate $\mathrm{D}-\mathrm{MCI}$ from $\mathrm{nD}-\mathrm{MCI}$, we computed the coupling between the FCD and ALFF across subjects in each group. Based on the two-sample $t$-test results of ALFF and FCD, subject-specific ALFF and FCD values were first extracted from the abnormal brain regions, respectively. Then, we performed a voxel-by-voxel Pearson correlation analysis between ALFF and FCD values in regions with alterations (between amplitude and FC) in each group, respectively.

\section{Results}

3.1. Neuropsychological Results. Age $(t=0.898, P=0.376)$, sex distribution $\left(\chi^{2}=0.161, P=0.735\right)$, and years of education $(t=0.464, P=0.645)$ did not differ between the two groups.
TABle 1: Demographics and neuropsychological data.

\begin{tabular}{lcccc}
\hline & $\begin{array}{c}\text { D-MCI } \\
\text { group }\end{array}$ & $\begin{array}{c}\text { nD-MCI } \\
\text { group }\end{array}$ & $t / \chi^{2}$ & $P$ value \\
\hline Gender, $n(\mathrm{M} / \mathrm{F})$ & $16(6 / 10)$ & $18(7 / 11)$ & 0.007 & 1.000 \\
Age, years & $69.6 \pm 6.2$ & $72.1 \pm 9.7$ & 0.898 & 0.376 \\
Education, years & $8.3 \pm 2.1$ & $8.5 \pm 1.8$ & 0.464 & 0.645 \\
MMSE & $26.6 \pm 1.1$ & $26.6 \pm 1.0$ & -0.037 & 0.971 \\
HAMD & $11.7 \pm 3.1$ & 0 & 16.0652 & 0.000 \\
D-NPI & $7.19 \pm 2.3$ & 0 & 13.3614 & 0.000 \\
\hline
\end{tabular}

Data represent mean \pm SD. Data were analysed using independent-samples $t$-tests. D-MCI: mild cognitive impairment with depression; $\mathrm{nD}-\mathrm{MCI}$ : nondepressed mild cognitive impairment; M: male; F: female; MMSE: Mini-Mental State Examination; D-NPI: depression domain on the Neuropsychiatric Inventory; HAMD: Hamilton Depression Rating Scale.

Detailed demographics and the corresponding statistical tests are presented in Table 1 .

3.2. ALFF Results. The two-sample $t$-tests revealed several related brain regions in which the ALFF values differed significantly between the D-MCI and $\mathrm{nD}-\mathrm{MCI}$ groups $(P<0.005$, 3dClustSim-corrected; Table 2$)$. In particular, we found that the D-MCI group exhibited significantly higher ALFF values than the $\mathrm{nD}$-MCI group in the right precentral cortex and significantly lower ALFF values in the left medial prefrontal cortex (Figure 1).

3.3. FCD Results. Similar to the ALFF values, the two-sample $t$-tests revealed significant differences in FCD between the DMCI and nD-MCI groups $(P<0.005,3$ dClustSim-corrected; Table 2). Specifically, we found that FCD values in the right middle temporal gyrus were greater in those with D-MCI than in those with $\mathrm{nD}-\mathrm{MCI}$ (Figure 1).

3.4. Altered Coupling between ALFF and FCD. Correlation analyses between the abnormal ALFF and FCD brain regions revealed a negative correlation between $\mathrm{MPFC}$ and $\mathrm{MTG}$ coupling in the nD-MCI (Figure 2) but not in the D-MCI group. Thus, for patients with $\mathrm{nD}-\mathrm{MCI}$, coupling was high in the $\mathrm{mPFC}$ when it was low in the MTG and vice versa.

\section{Discussion}

Here, we investigated alterations in ALFF-FCD and coupling of ALFF with FCD in patients with D-MCI and nD-MCI. The D-MCI group exhibited significantly higher FCD in the right MTG, significantly higher ALFF in the left $\mathrm{mPFC}$, and significantly lower ALFF in the right precentral gyrus (preCG) than the $\mathrm{nD}$-MCI group. We also found a negative correlation between ALFF-FCD coupling in the mPFC and the MTG but only in patients with D-MCI.

The mPFC is an important node in the cortico-striatopallido-thalamic loops and in the medial network, contributing to emotional processes and regulation [27]. Several neuroimaging studies have demonstrated that the $\mathrm{mPFC}$ is a key brain region in depressive symptomatology. Xie et al. [6] reported that depressive symptoms in MCI are related to grey matter volume loss in several brain regions, including the mPFC, posterior MTG, and insula. Furthermore, Sacuiu 
TABLE 2: Brain regions with significantly lower ALFF-FCD values in the $\mathrm{D}-\mathrm{MCI}$ group than in the $\mathrm{nD}-\mathrm{MCI}$ group.

\begin{tabular}{|c|c|c|c|c|c|c|}
\hline \multirow{2}{*}{ Brain regions } & \multirow{2}{*}{ Voxels } & \multirow[t]{2}{*}{ BA } & \multicolumn{3}{|c|}{$\begin{array}{c}\text { MNI } \\
\text { coordinates }\end{array}$} & \multirow[t]{2}{*}{$t$ value } \\
\hline & & & $X$ & $Y$ & $Z$ & \\
\hline \multicolumn{7}{|l|}{$A L F F$} \\
\hline Prefrontal_medial_L & 32 & 10 & -9 & 63 & 27 & 4.6674 \\
\hline Precentral_R & 28 & 6 & 27 & -3 & 51 & -5.7117 \\
\hline \multicolumn{7}{|l|}{$F C D$} \\
\hline Temporal_mid_R & 117 & 37 & 51 & -66 & 6 & 4.9394 \\
\hline
\end{tabular}

et al. and Gonzales et al. reported that D-MCI patients have increased cortical atrophy in the anterior cingulate and the frontal lobe, and decreased left mPFC thickness was associated with increased negative affect $[7,8]$. Other studies have also demonstrated that depressed individuals with MCI had more white matter atrophy in the frontal, parietal, and temporal cortices than those with no-symptom MCI did [9-11]. Based on R-fMRI and ALFF analyses, Wang et al. [28] found that MDD patients had abnormal ALFF values in the $\mathrm{mPFC}$, precentral gyrus, and other regions. Using magnetic resonance spectroscopy, McEwen et al. [29] reported that glutamate levels in the mPFC were higher in women with postpartum depression than in controls. Savitz et al. [30] found that dysfunction of the serotonin-1A receptor in the $\mathrm{mPFC}$ might play a role in the genesis of MDD. Additionally, a recent animal study [31] suggested that synaptogenic activity in the mPFC is associated with a rapid antidepressant response to ketamine. Thus, our results are in line with previous findings, suggesting that the altered spontaneous ALFF values in the mPFC might be a characteristic of the neurological impairments of MCI with depressive symptoms.

A neuroimaging study reported that the preCG was involved in MDD [32]. Peng et al. [33] observed decreased cortical thickness in the preCG in patients with untreated first-episode MDD. Using voxel-based morphometry, Taki et al. [34] found that male patients with subthreshold depression had significantly smaller right preCG volumes than healthy controls. Several studies have also demonstrated that grey matter structural changes in the right preCG can predict the subsequent onset of MDD and pose an increased risk for mood disorders [35-37]. A study by Ho et al. [38] revealed that the symptom of alexithymia that occurs in MDD was associated with reduced functional connectivity in the right preCG and several other right hemisphere regions that are associated with cognitive regulation in the default mode network (DMN). They found that subjective pleasantness was related to the preCG, right cerebellum, and right inferior frontal gyrus [39]. The preCG is located at the primary motor cortex, which is required for the initiation of purposeful movements via integration of information sent from the sensorimotor cortex [40]. Several studies have confirmed the association between psychomotor retardation, poor action planning, and alterations in the preCG [41, 42]. Therefore, the abnormal ALFF in the preCG of our study might be related to the depressive symptoms in our D-MCI patients.

MTG is an important node within the DMN and the affective network (AN), which are involved in selfreferential processing, emotion, and regulation [43-45]. Neuroimaging evidence has demonstrated that altered FC occurs in MDD patients between the MTG and other nodes within the DMN and AN. For the FC of the right MTG, Ma et al. [46] found that patients with treatment-resistant depression as well as those with treatment-responsive depression showed abnormal connectivity mainly in the DMN. Based on FC analyses of the subgenual anterior cingulate cortex (sgACC), MDD patients showed disruptions in FC between the sgACC and MTG [47]. Du et al. [48] investigated the brain-circuit mechanisms of suicidal ideation (SI) in MDD, finding that the SI group exhibited decreased intrinsic FC among the rostral anterior cingulate cortex (rACC), the orbitomedial prefrontal cortex, and the right MTG compared with healthy controls and those with MDD without SI. In the SI group, the FC strength between the right rACC and the MTG positively correlated with SI severity. The altered FCD in the MTG in the present study expands the knowledge gained from previous studies of abnormal FC in MDD patients by investigating the FC in the right MTG. MDD is characterised by the presence of negative thoughts about oneself, the world, or the future. We hypothesised that the altered FC in the right MTG might contribute to the negative thoughts and negative emotions observed in patients with MCI and depressive symptoms.

Interestingly, we found a negative correlation between ALFF-FCD coupling in the MPFC and the MTG but only in the $\mathrm{nD}-\mathrm{MCI}$ group. De Bellis and Hooper [49] reported that maltreated youth with depressive disorders had significantly weaker activation of the mPFC in response to attentional targets and stronger activation in the MTG in response to sad distracters during an emotional oddball task than controls. They concluded that maltreated youth with depressive disorders had dysfunctional cognitive and emotional processing because of the $\mathrm{mPFC}$ involvement in cognitive control circuits and the MTG involvement in ventral emotional circuits. Du et al. [48] also reported that depressed patients with suicidal ideation had decreased intrinsic FC between the MPFC and the right MTG, and they suggested that the disrupted frontolimbic circuits might impact decision-making and emotional processing in depressed patients with suicidal ideation. The mPFC and right MTG are two nodes within the DMN. According to previous neuroimaging studies, the DMN can be generally divided into anterior $(\mathrm{aDMN})$ and posterior (pDMN) subnetworks. The former is related to self-referential thought, and the latter is related to episodic memory retrieval and scene construction [50-52]. The aDMN mostly consists of the mPFC, anterior cingulate cortex, anterior temporal lobe, and inferior frontal gyrus, whereas the pDMN mostly contains the posterior cingulate cortex (PCC), precuneus, angular gyrus, hippocampus, and temporal lobe. A recent fMRI study revealed that the activation within these subsystems and the connectivity between the aDMN and pDMN contribute differently to 


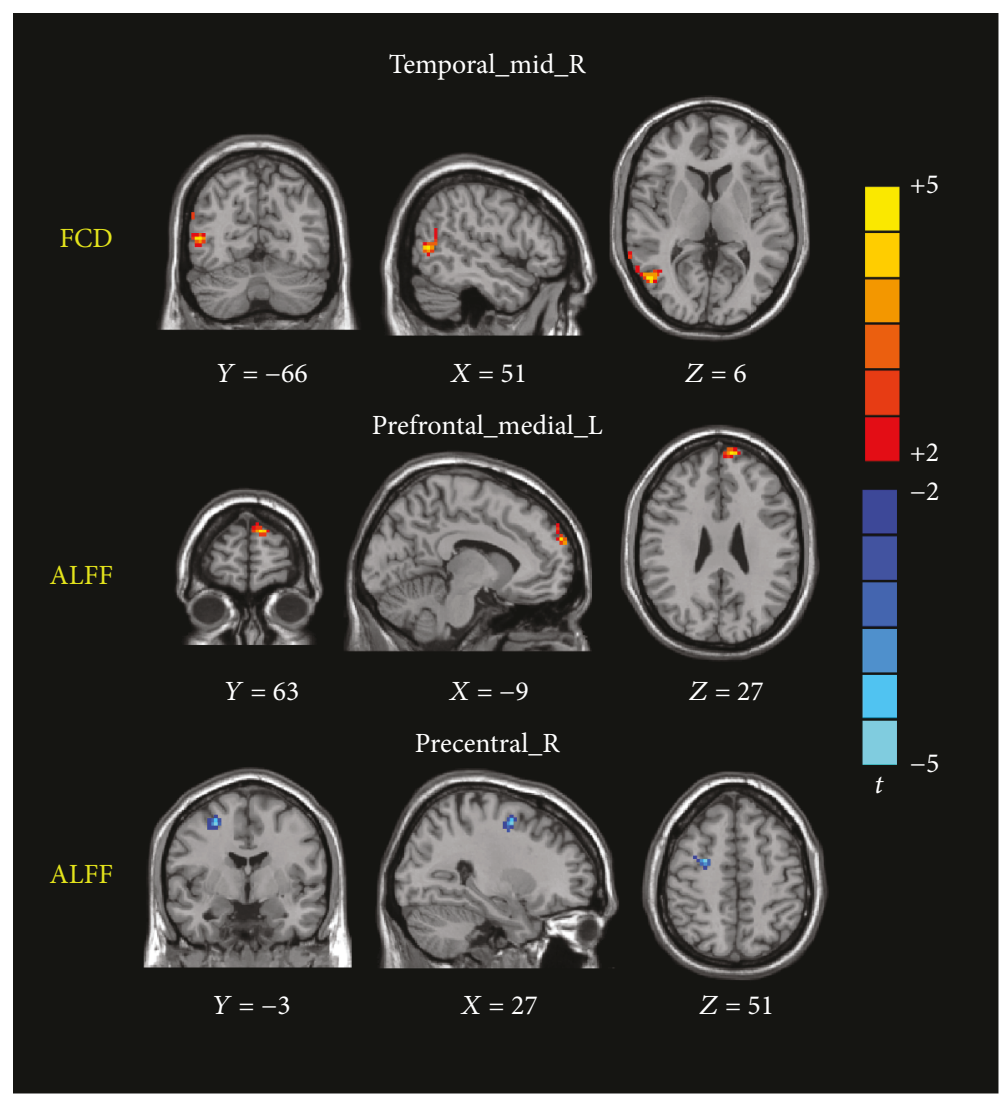

FIGURE 1: Brain regions showing differences in FCD or ALFF values between the D-MCI and nD-MCI groups (contrast $=\mathrm{D}-\mathrm{MCI}-\mathrm{nD}-\mathrm{MCI}$ ).

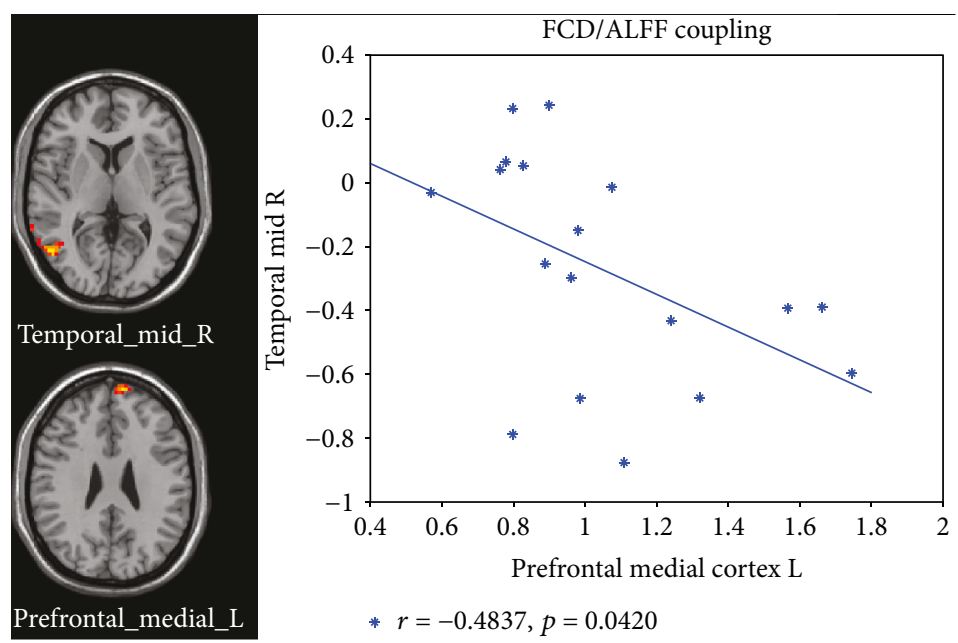

FIGURE 2: Significant correlations between FCD and ALFF in the $\mathrm{nD}-\mathrm{MCI}$ brain. FCD values of the right temporal middle gyrus and ALFF values of the medial prefrontal cortex were subtracted from the abnormal brain regions resulting from $t$-contrast $\mathrm{nD}$-MCI versus $\mathrm{D}$-MCI.

future-oriented thoughts [53]. How the brain creates emotions is complex and esoteric. Using a psychological constructionist approach, Lindquist et al. hypothesised that several brain regions, including the $\mathrm{mPFC}$, medial temporal lobe (MTL), and PCC, are important for realising instances of emotional experiences and perceptions [54]. The negative correlation in ALFF-FCD coupling between the mPFC and the MTG in our study might contribute to decision-making, future-oriented thoughts, and experiences and perceptions of emotion in MCI with depressive symptoms.

This study had several potential limitations. First, the sample size was relatively small, which might have resulted in low statistical power and the absence of correlations between FCD/ALFF and physiological measurements. Second, previous studies have suggested that neuronal oscillations in various frequency intervals have different specific 
properties and physiological functions [14]. While short- and long-range FCD have been used in fMRI studies [55], further investigations into coupling between long- or short-range FCD with ALFF at various frequency intervals should be performed. Finally, the choice of a clinical assessment scale is very important. In our study, we selected the HAMD and the NPI to evaluate depressive symptoms in MCI, as a previous study did [9]. However, other studies have used different scales, such as the Geriatric Depression Scale [56], the Cornell Scale for Depression in Dementia [57], and the Center for Epidemiologic Studies Depression Scale [58]. Evaluating depressive symptoms comprehensively and accurately in MCI is difficult because of the cognitive impairment. Therefore, in future studies, we should evaluate clinical depressive symptoms with multiple scales, based on the characteristics of each scale.

\section{Conclusions}

In the current study, we observed differences between DMCI and $\mathrm{nD}-\mathrm{MCI}$ in terms of FCD and ALFF values that were derived from R-fMRI. We also examined differences in ALFF-FCD coupling in D-MCI and $\mathrm{nD}-\mathrm{MCI}$. The findings indicated that pathological factors lead to dysfunctional ALFF-FCD coupling between the MPFC and the MTG in $\mathrm{D}-\mathrm{MCI}$. Investigation of imaging coupling provides a synergistic approach to unravelling the features of the functional changes in MCI and provides a new insight into the underlying neural mechanism of MCI with depressive symptoms.

\section{Conflicts of Interest}

The authors declare no conflict of interest.

\section{Acknowledgments}

This research was supported by the Doctoral Scientific Research Foundation of the Second Affiliated Hospital, Wenzhou Medical University, the General Project of the Department of Science and Technology of Zhejiang Province (2017KY109) (to Xiaozheng Liu), the Public Project of the Department of Science and Technology of Wen Zhou (H20160002) (to Bangli Shen), and the General Project of the Department of Science and Technology of Zhejiang Province (2013KYB065, 2018KY031) (to Zhongwei Guo).

\section{References}

[1] J. D. Grill, L. G. Apostolova, S. Bullain et al., "Communicating mild cognitive impairment diagnoses with and without amyloid imaging," Alzheimer's Research \& Therapy, vol. 9, no. 1, p. 35, 2017.

[2] R. C. Petersen, R. O. Roberts, D. S. Knopman et al., "Mild cognitive impairment: ten years later," Archives of Neurology, vol. 66 , no. 12, pp. 1447-1455, 2009.

[3] Z. Ismail, H. Elbayoumi, C. E. Fischer et al., "Prevalence of depression in patients with mild cognitive impairment: a systematic review and meta-analysis," JAMA Psychiatry, vol. 74, no. 1, pp. 58-67, 2017.
[4] Y. Gao, C. Huang, K. Zhao et al., "Retracted: depression as a risk factor for dementia and mild cognitive impairment: a meta-analysis of longitudinal studies," International Journal of Geriatric Psychiatry, vol. 28, no. 5, pp. 441-449, 2013.

[5] R. J. Mourao, G. Mansur, L. F. Malloy-Diniz, E. C. Costa, and B. S. Diniz, "Depressive symptoms increase the risk of progression to dementia in subjects with mild cognitive impairment: systematic review and meta-analysis," International Journal of Geriatric Psychiatry, vol. 31, no. 8, pp. 905-911, 2016.

[6] C. Xie, W. Li, G. Chen et al., "The co-existence of geriatric depression and amnestic mild cognitive impairment detrimentally affect gray matter volumes: voxel-based morphometry study," Behavioural Brain Research, vol. 235, no. 2, pp. 244-250, 2012.

[7] S. Sacuiu, P. S. Insel, S. Mueller et al., "Chronic depressive symptomatology in mild cognitive impairment is associated with frontal atrophy rate which hastens conversion to Alzheimer dementia," The American Journal of Geriatric Psychiatry, vol. 24, no. 2, pp. 126-135, 2016.

[8] M. M. Gonzales, P. S. Insel, C. Nelson et al., "Cortical atrophy is associated with accelerated cognitive decline in mild cognitive impairment with subsyndromal depression," The American Journal of Geriatric Psychiatry, vol. 25, no. 9, pp. 980-991, 2017.

[9] G. J. Lee, P. H. Lu, X. Hua et al., "Depressive symptoms in mild cognitive impairment predict greater atrophy in Alzheimer's disease-related regions," Biological Psychiatry, vol. 71, no. 9, pp. 814-821, 2011.

[10] R. S. Mackin, P. Insel, D. Tosun et al., "The effect of subsyndromal symptoms of depression and white matter lesions on disability for individuals with mild cognitive impairment," The American Journal of Geriatric Psychiatry, vol. 21, no. 9, pp. 906-914, 2013.

[11] W. Li, L. T. Muftuler, G. Chen et al., "Effects of the coexistence of late-life depression and mild cognitive impairment on white matter microstructure," Journal of the Neurological Sciences, vol. 338, no. 1-2, pp. 46-56, 2014.

[12] L. J. Zheng, G. F. Yang, X. Y. Zhang et al., "Altered amygdala and hippocampus effective connectivity in mild cognitive impairment patients with depression: a resting-state functional MR imaging study with granger causality analysis," Oncotarget, vol. 8, no. 15, pp. 25021-25031, 2017.

[13] C. Xie, J. Goveas, Z. Wu et al., "Neural basis of the association between depressive symptoms and memory deficits in nondemented subjects: resting-state fMRI study," Human Brain Mapping, vol. 33, no. 6, pp. 1352-1363, 2012.

[14] Y. Li, B. Jing, H. Liu et al., "Frequency-dependent changes in the amplitude of low-frequency fluctuations in mild cognitive impairment with mild depression," Journal of Alzheimer's Disease, vol. 58, no. 4, pp. 1175-1187, 2017.

[15] D. Mascali, M. DiNuzzo, T. Gili et al., "Intrinsic patterns of coupling between correlation and amplitude of lowfrequency fMRI fluctuations are disrupted in degenerative dementia mainly due to functional disconnection," PLoS One, vol. 10, no. 4, article e0120988, 2015.

[16] Z. Zhang, Q. Xu, W. Liao et al., "Pathological uncoupling between amplitude and connectivity of brain fluctuations in epilepsy," Human Brain Mapping, vol. 36, no. 7, pp. 27562766, 2015.

[17] X. D. Zhang, X. L. Jiang, Z. Cheng et al., "Decreased coupling between functional connectivity density and amplitude of low frequency fluctuation in non-neuropsychiatric systemic 
lupus erythematosus: a resting-stage functional MRI study," Molecular Neurobiology, vol. 54, no. 7, pp. 5225-5235, 2017.

[18] B. Zhang, M. Li, W. Qin et al., "Altered functional connectivity density in major depressive disorder at rest," European Archives of Psychiatry and Clinical Neuroscience, vol. 266, no. 3, pp. 239-248, 2016.

[19] R. C. Petersen, G. E. Smith, S. C. Waring, R. J. Ivnik, E. G. Tangalos, and E. Kokmen, "Mild cognitive impairment: clinical characterization and outcome," Archives of Neurology, vol. 56, no. 3, pp. 303-308, 1999.

[20] A. Gmitrowicz and A. Kucharska, "Developmental disorders in the fourth edition of the American classification: diagnostic and statistical manual of mental disorders (DSM IV - optional book)," Psychiatria Polska, vol. 28, no. 5, pp. 509-521, 1994.

[21] M. Hamilton, "Development of a rating scale for primary depressive illness," British Journal of Social and Clinical Psychology, vol. 6, no. 4, pp. 278-296, 1967.

[22] J. L. Cummings, M. Mega, K. Gray, S. Rosenberg-Thompson, D. A. Carusi, and J. Gornbein, "The Neuropsychiatric Inventory: comprehensive assessment of psychopathology in dementia," Neurology, vol. 44, no. 12, pp. 2308-2314, 1994.

[23] L. S. Schneider, P. N. Tariot, C. G. Lyketsos et al., "National Institute of Mental Health Clinical Antipsychotic Trials of Intervention Effectiveness (CATIE): Alzheimer disease trial methodology," The American Journal of Geriatric Psychiatry, vol. 9, no. 4, pp. 346-360, 2001.

[24] Y. F. Zang, Y. He, C. Z. Zhu et al., "Altered baseline brain activity in children with ADHD revealed by resting-state functional MRI," Brain \& Development, vol. 29, no. 2, pp. 83-91, 2007.

[25] R. L. Buckner, J. Sepulcre, T. Talukdar et al., "Cortical hubs revealed by intrinsic functional connectivity: mapping, assessment of stability, and relation to Alzheimer's disease," The Journal of Neuroscience, vol. 29, no. 6, pp. 1860-1873, 2009.

[26] A. Ledberg, S. Akerman, and P. E. Roland, "Estimation of the probabilities of 3D clusters in functional brain images," NeuroImage, vol. 8, no. 2, pp. 113-128, 1998.

[27] J. L. Price and W. C. Drevets, "Neurocircuitry of mood disorders," Neuropsychopharmacology, vol. 35, no. 1, pp. 192-216, 2010.

[28] L. Wang, Q. Kong, K. Li et al., "Frequency-dependent changes in amplitude of low-frequency oscillations in depression: a resting-state fMRI study," Neuroscience Letters, vol. 614, pp. 105-111, 2016.

[29] A. M. McEwen, D. T. A. Burgess, C. C. Hanstock et al., "Increased glutamate levels in the medial prefrontal cortex in patients with postpartum depression," Neuropsychopharmacology, vol. 37, no. 11, pp. 2428-2435, 2012.

[30] J. Savitz, I. Lucki, and W. C. Drevets, " $5-\mathrm{HT}_{1 \mathrm{~A}}$ receptor function in major depressive disorder," Progress in Neurobiology, vol. 88, no. 1, pp. 17-31, 2009.

[31] N. Li, B. Lee, R. J. Liu et al., "mTOR-dependent synapse formation underlies the rapid antidepressant effects of NMDA antagonists," Science, vol. 329, no. 5994, pp. 959-964, 2010.

[32] X. L. Wang, M. Y. du, T. L. Chen et al., "Neural correlates during working memory processing in major depressive disorder," Progress in Neuro-Psychopharmacology \& Biological Psychiatry, vol. 56, pp. 101-108, 2015.

[33] D. Peng, F. Shi, G. Li et al., "Correction: surface vulnerability of cerebral cortex to major depressive disorder," PLoS One, vol. 10, no. 6, article e0128947, 2015.
[34] Y. Taki, S. Kinomura, S. Awata et al., "Male elderly subthreshold depression patients have smaller volume of medial part of prefrontal cortex and precentral gyrus compared with agematched normal subjects: a voxel-based morphometry," Journal of Affective Disorders, vol. 88, no. 3, pp. 313-320, 2005.

[35] L. C. Foland-Ross, M. D. Sacchet, G. Prasad, B. Gilbert, P. M. Thompson, and I. H. Gotlib, "Cortical thickness predicts the first onset of major depression in adolescence," International Journal of Developmental Neuroscience, vol. 46, pp. 125-131, 2015.

[36] M. Papmeyer, S. Giles, J. E. Sussmann et al., "Cortical thickness in individuals at high familial risk of mood disorders as they develop major depressive disorder," Biological Psychiatry, vol. 78, no. 1, pp. 58-66, 2015.

[37] X. Zhang, S. Yao, X. Zhu, X. Wang, X. Zhu, and M. Zhong, "Gray matter volume abnormalities in individuals with cognitive vulnerability to depression: a voxel-based morphometry study," Journal of Affective Disorders, vol. 136, no. 3, pp. 443-452, 2012.

[38] N. S. P. Ho, M. M. C. Wong, and T. M. C. Lee, "Neural connectivity of alexithymia: specific association with major depressive disorder," Journal of Affective Disorders, vol. 193, pp. 362-372, 2016.

[39] S. Kühn and J. Gallinat, "The neural correlates of subjective pleasantness," NeuroImage, vol. 61, no. 1, pp. 289-294, 2012.

[40] V. Taschereau-Dumouchel and S. Hetu, "Visuomotor representations within the human primary motor cortex: the elusive markers of visuomotor associative learning," The Journal of Neuroscience, vol. 32, no. 3, pp. 759-760, 2012.

[41] S. Walther, S. Hügli, O. Höfle et al., "Frontal white matter integrity is related to psychomotor retardation in major depression," Neurobiology of Disease, vol. 47, no. 1 , pp. 13-19, 2012.

[42] J. S. Buyukdura, S. M. McClintock, and P. E. Croarkin, "Psychomotor retardation in depression: biological underpinnings, measurement, and treatment," Progress in NeuroPsychopharmacology \& Biological Psychiatry, vol. 35, no. 2, pp. 395-409, 2011.

[43] G. Northoff and F. Bermpohl, "Cortical midline structures and the self," Trends in Cognitive Sciences, vol. 8, no. 3, pp. 102-107, 2004.

[44] R. N. Spreng, R. A. Mar, and A. S. N. Kim, “The common neural basis of autobiographical memory, prospection, navigation, theory of mind, and the default mode: a quantitative metaanalysis," Journal of Cognitive Neuroscience, vol. 21, no. 3, pp. 489-510, 2009.

[45] L.-L. Zeng, H. Shen, L. Liu et al., "Identifying major depression using whole brain functional connectivity: a multivariate pattern analysis," Brain, vol. 135, no. 5, pp. 1498-1507, 2012.

[46] C. Ma, J. Ding, J. Li et al., "Resting-state functional connectivity bias of middle temporal gyrus and caudate with altered gray matter volume in major depression," PLoS One, vol. 7, no. 9, article e45263, 2012.

[47] H. Wu, H. Sun, J. Xu et al., "Changed hub and corresponding functional connectivity of subgenual anterior cingulate cortex in major depressive disorder," Frontiers in Neuroanatomy, vol. 10, p. 120, 2016.

[48] L. Du, J. Zeng, H. Liu et al., "Fronto-limbic disconnection in depressed patients with suicidal ideation: a resting-state functional connectivity study," Journal of Affective Disorders, vol. 215, pp. 213-217, 2017. 
[49] M. D. De Bellis and S. R. Hooper, "Neural substrates for processing task-irrelevant emotional distracters in maltreated adolescents with depressive disorders: a pilot study," Journal of Traumatic Stress, vol. 25, no. 2, pp. 198-202, 2012.

[50] J. S. Damoiseaux, C. F. Beckmann, E. J. S. Arigita et al., "Reduced resting-state brain activity in the "default network" in normal aging," Cerebral Cortex, vol. 18, no. 8, pp. 18561864, 2008.

[51] X. Lei, Z. Zhao, and H. Chen, "Extraversion is encoded by scale-free dynamics of default mode network," NeuroImage, vol. 74, pp. 52-57, 2013.

[52] X. Lei, Y. Wang, H. Yuan, and D. Mantini, "Neuronal oscillations and functional interactions between resting state networks," Human Brain Mapping, vol. 35, no. 7, pp. 35173528, 2014.

[53] X. Xu, H. Yuan, and X. Lei, "Activation and connectivity within the default mode network contribute independently to future-oriented thought," Scientific Reports, vol. 6, no. 1, article 21001, 2016.

[54] K. A. Lindquist, T. D. Wager, H. Kober, E. Bliss-Moreau, and L. F. Barrett, "The brain basis of emotion: a meta-analytic review," Behavioral and Brain Sciences, vol. 35, no. 3, pp. 121-143, 2012.

[55] W. Qin, Y. Xuan, Y. Liu, T. Jiang, and C. Yu, "Functional connectivity density in congenitally and late blind subjects," Cerebral Cortex, vol. 25, no. 9, pp. 2507-2516, 2015.

[56] C. M. Sarabia-Cobo, B. García-Rodríguez, M. J. Navas, and H. Ellgring, "Emotional processing in patients with mild cognitive impairment: the influence of the valence and intensity of emotional stimuli: the valence and intensity of emotional stimuli influence emotional processing in patients with mild cognitive impairment," Journal of the Neurological Sciences, vol. 357, no. 1-2, pp. 222-228, 2015.

[57] M. L. Barca, K. Persson, R. Eldholm et al., "Trajectories of depressive symptoms and their relationship to the progression of dementia," Journal of Affective Disorders, vol. 222, pp. 146$152,2017$.

[58] C. L. Li, Y. C. Chiu, Y. B. Bai, J. D. Lin, F. Stanaway, and H. Y. Chang, "The co-occurrence of depressive symptoms and cognitive impairment and its relationship with selfcare behaviors among community dwelling older adults with diabetes," Diabetes Research and Clinical Practice, vol. 129, pp. 73-78, 2017. 


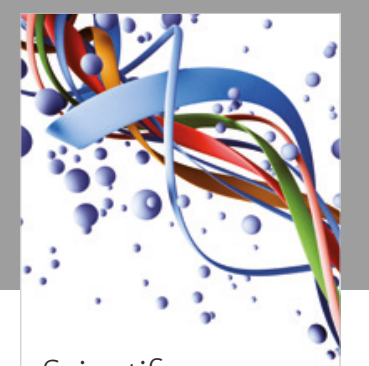

Scientifica
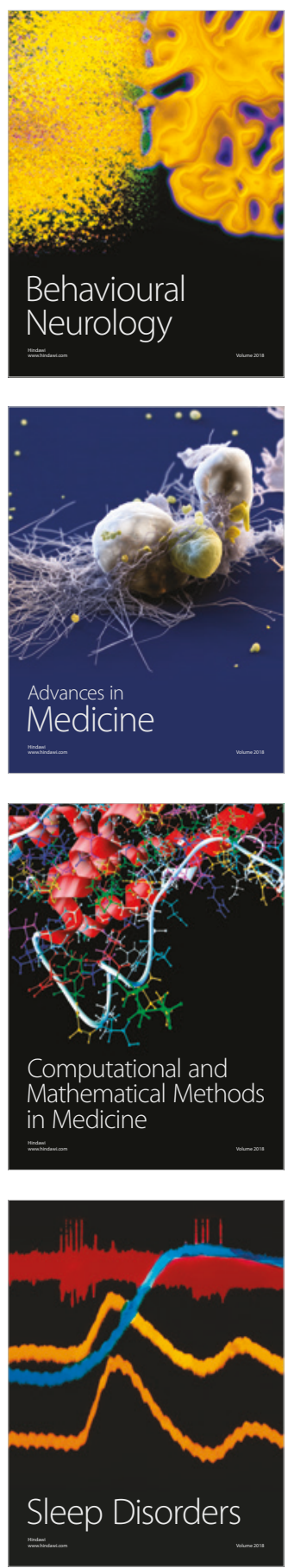

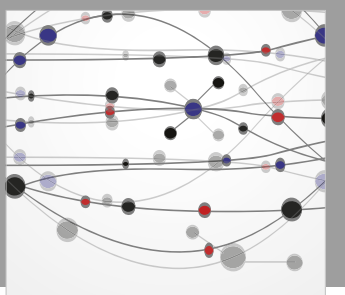

The Scientific World Journal

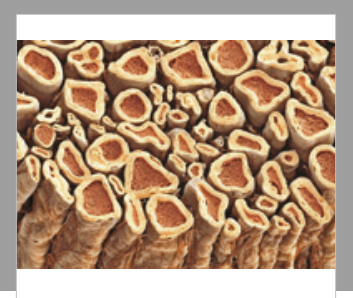

Case Reports in

Neurological Medicine

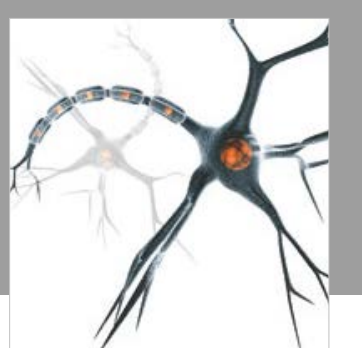

Neural Plasticity

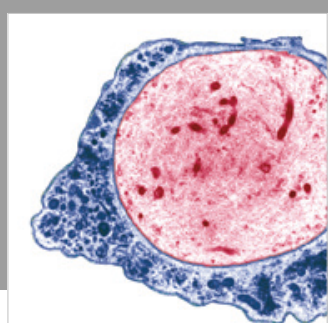

Multiple Sclerosis

International

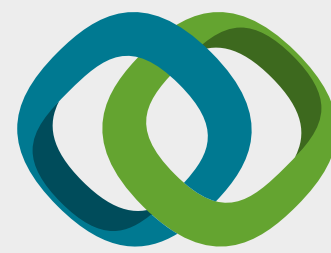

Hindawi

Submit your manuscripts at

www.hindawi.com
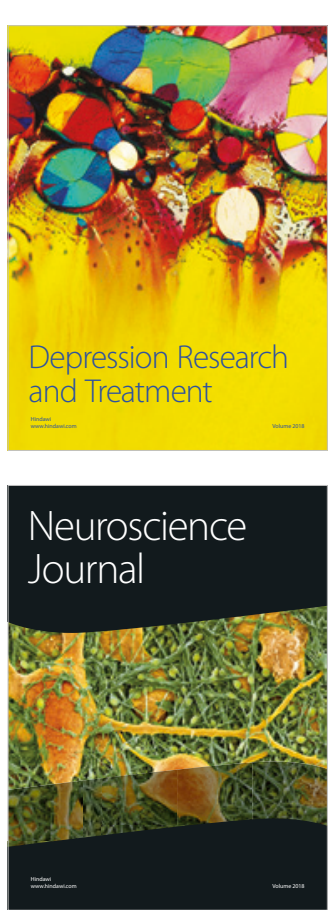

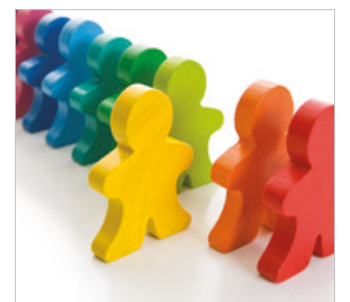

Autism

Research and Treatment
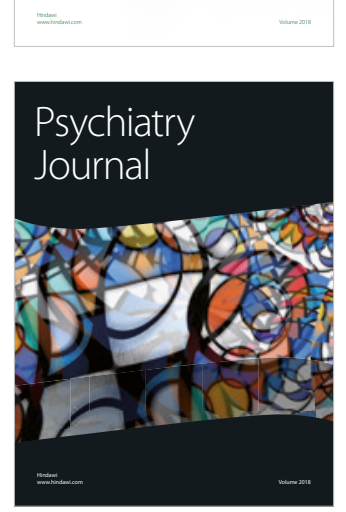
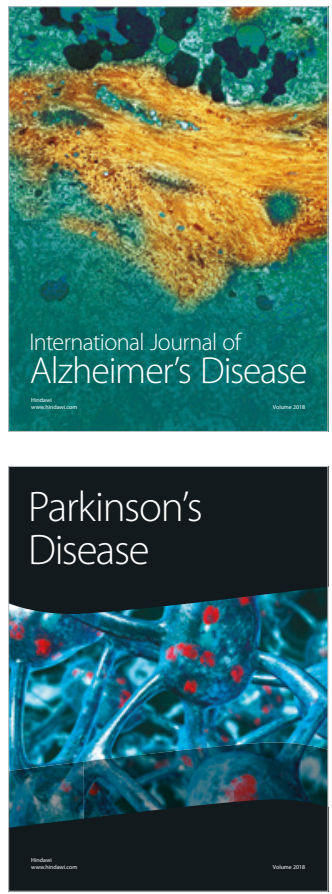
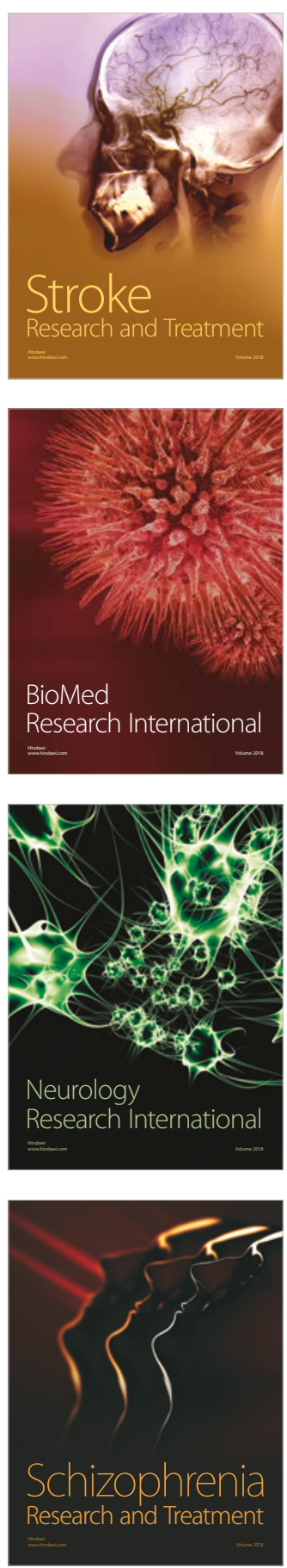